$\infty$

CHOICE

Postgraduate Medical School, University of Surrey and Department of Histopathology, Royal Surrey County Hospital, Guildford, Surrey, UK

Correspondence to: Professor M G Cook, Royal Surrey County Hospital, Egerton Road, Guildford, Surrey GU2 7XX,UK; m.cook@nhs.net

Accepted 5 May 2008

Published Online First

30 May 2008

\title{
Pathology of sentinel lymph nodes for melanoma
}

\author{
M G Cook, S Di Palma
}

\section{ABSTRACT}

As a concept sentinel lymph node biopsy seems attractive in that it attempts to identify the first lymph node, rather than the nearest node, draining a particular anatomic area where a tumour has arisen. Pathological assessment can then indicate whether metastases are present and the procedure is either a strong prognostic indicator or possibly therapeutic in itself. These comments apply to any tumour type, but with melanoma the pathological procedure is more problematic and any benefits above prognosis and staging are not universally accepted. The procedure does give accurate staging without the extra morbidity of regional node dissection and many patients gain psychological support from the information gained.

\section{POTENTIAL BENEFITS OF SENTINEL LYMPH NODE BIOPSY FOR MELANOMA}

Excision of the sentinel lymph node (SLN) enables a prognostic assessment to be made that is more powerful than thickness measurements on the primary. ${ }^{12}$ This in itself is sufficient, to many, to justify the procedure since it has low morbidity. ${ }^{3}$ The SLN status is an important criterion for entry into clinical trials and therefore until a clear effective systemic therapy is established SLN biopsy (SLNB) will have importance and the SLN status may be the threshold to entry into a treatment programme.

The initial reason for development of SLNB was to enable selection of patients more likely to receive benefits from regional node dissection (RND). It had already been shown that RND was not effective on unselected patients but in the absence of alternatives the surgical treatment option was retained. The SLNB seemed a way of directing RND to a group of patients more likely to benefit from wide excision of nodes. This remains the usual procedure, although evolution towards offering RND to an even more restricted group of patients is proceeding. Whether SLNB by itself is of therapeutic benefit is not yet established. There is a suggestion of prolongation of disease-free survival for SLNB patients as compared with observation followed by RND only when clinical evidence of metastases develops. Prolongation of overall survival has not yet been demonstrated but longer follow-up may reveal this. ${ }^{2}$

\section{POTENTIAL PROBLEMS OF SLNB}

Any disadvantages of SLNB are also not established. The anxieties about an increase in "in transit" metastase ${ }^{45}$ have not been upheld. ${ }^{67}$ The theoretical concern about removal of what is likely to be the first line of immunological defence still requires further study. A suggested alternative procedure is high-grade ultrasound, ${ }^{8}$ but $4.5 \mathrm{~mm}$ is the smallest size that can be consistently detected with this technique and the majority of metastases in SLN for melanoma are smaller than that and therefore this alternative does not appear acceptable as a substitute for SLNB. The SLNB procedure has some associated morbidity but this is much less than for RND, and at least $70 \%$ of patients with melanoma will avoid this morbidity by having the lesser procedure. The National Institute for Health and Clinical Excellence (NICE) report on skin tumours ${ }^{9}$ has concluded that SLNB has merit for staging, and that it should continue to be done in existing centres as part of clinical trials, but has not wholeheartedly recommended SLNB for all melanoma. This recommendation is in spite of the current paucity of suitable trials into which to enter patients. The NICE advice may need modification in the light of the recent report of MSLT-1 since some prolongation of disease-free survival has been demonstrated ${ }^{2}$ and improved overall survival cannot be ruled out.

\section{SURGICAL PROCEDURES EFFECTING PATHOLOGICAL ASSESSIMENT}

The identification of the SLN by the surgeon should be based on preoperative lymphoscintigraphy and intraoperative injection of vital blue dye and radiocolloids. The lymphoscintigraphy demonstrates the direction of the lymph drainage from any cutaneous site and enables the first or sentinel lymph nodes in each basin to be identified and marked on the overlying skin. There is some variation in definitions of SLN and so there may be variation in the numbers of SLN excised between surgeons or between centres.

The SLN should be excised with a cuff of fat and without trauma to the node. Examination of the subcapsular zone of the SLN is most likely to reveal metastases and so it is important that this should not be damaged by the surgery. ${ }^{10}$ Confirmation of what is a true SLN may be obtained by using carbon dye injected at the primary site. This tends to be localised in the area most likely to contain metastases and its presence confirms the node as a true SLN. Unfortunately this carbon preparation is not available in all countries. Where the surgeon sees the afferent lymphatics with the blue dye he can use a ligature to identify this aspect of the node so that the pathologist is aware of the orientation of the node, and this therefore facilitates the slicing through the median plane.

\section{HISTOPATHOLOGICAL AND PATHOBIOLOGICAL PECULIARITIES OF MELANOCYTIC TUMOURS AFFECTING INTERPRETATION OF SLN}

In contrast to interpretation of SLN for other malignancies, melanoma presents extra difficulties due to its tendency to metastasise in small groups 
or even single cells, which on conventional H\&E staining are similar to other cells normally present in lymph nodes at least at scanning magnification. The assessment of the lymph node must be designed to detect as many as possible of these metastases. To detect all of them would involve serial sectioning of the SLN to extinction resulting in thousands of sections per node. This is clearly not practicable and a balance between workload and efficiency is required and several procedures have been developed.

\section{PATHOLOGICAL PROCEDURES}

\section{Dissection}

Dissection of the SLN can be by random parallel "bread loaf" slicing ${ }^{11}$ or single slicing through the central plane (bivalve technique). ${ }^{12}$

Since Cochran, in an early study, ${ }^{13}$ showed that the majority of metastases are in a central plane (see fig 1), the bivalve technique, in which a slice is made through the convex capsule and the hilum to reveal the maximum cut surface area, has been adopted by us. $^{14}$ The alternative technique recommended by Starz et $a l^{11}$ consists of slicing the SLN at $2 \mathrm{~mm}$ intervals. It is attractive in that it does not depend on the slightly difficult identification of a central plane and also in that it does not require multiple sections on each slice. However, in larger nodes, there may be a need for several blocks.

On balance we have preferred to use the bivalving approach, but using some dissection of the lymph node from the surrounding fat followed by a combined visual assessment and palpation to identify the central plane through which the node is bisected.

\section{Sectioning and staining}

Further options exist in the sectioning and staining of the bivalved node. Cochran ${ }^{12}$ recommended serial sections from the two mid-plane surfaces stained with a combination of $\mathrm{H} \& \mathrm{E}$ and melanocyte markers (S100, HMB45, Melan A). Starz ${ }^{11}$ examined only two sections from each block dissected in a different way, and used $\mathrm{H} \& \mathrm{E}$ and immunohistochemistry. Both of these procedures detected metastases in $15-20 \%$ of patients.

Since immunohistochemistry increases the detection rate of metastases by at least $10 \%{ }^{15}$ we have used it from the beginning.

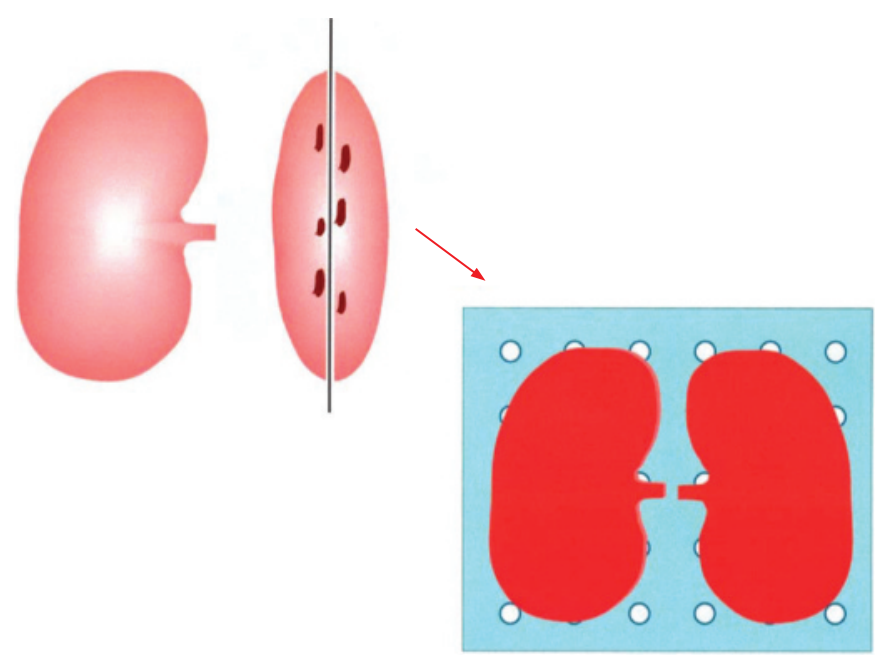

Figure 1 Metastases of melanoma are said to occur close to the median plane of the sentinel lymph node and therefore bivalving, as shown, and embedding face down in the cassette are recommended.
The particular antibody used depends on local preference. We have tried HMB45, MelanA, MiTF and S100. Melan A and HMB45 are widely used but they can be criticised as being less sensitive; however, on the other hand they have the advantage of being more specific. S100 has been chosen as the first-line antibody since it is the most sensitive, the least demanding technically, and the cheapest. However, it does require the pathologist to gain experience in its interpretation, particularly in distinguishing melanocytes from clumps of dendritic cells. We have overcome this rare problem by always having unstained adjacent sections for more specific antibodies in reserve for use in more problematic cases. We use Melan A most often for this confirmatory purpose. We have found HMB45 positivity in only $70 \%$ of melanomas and therefore do not regard it as sufficiently sensitive. A cocktail of antibodies including Melan A, HMB45, S100 and tyrosinase such as Pan Mel+ (Biocare Medical, Concord, California, USA) has high sensitivity and specificity but is more expensive and technically more demanding. With experience, S100 has the highest sensitivity and particularly in combination with diaminobenzidine chromogen even single cells can be identified on screening magnification and confirmed on the adjacent $\mathrm{H} \& \mathrm{E}$. This enables distinction from other potentially positive cells such as dendritic cells and nerves. We assess the same cells on immediately adjacent sections stained with $\mathrm{H} \& \mathrm{E}$. Unless we are convinced that the immunohistochemistry positivity corresponds with a cell with cytological features of a melanocyte, we do not regard the SLN as positive.

Melanophages may sometimes be identified without melanocytes in the SLN and it could be speculated that these features represent intranodal regression, but the melanophages could equally well represent drainage of melanophages from the primary melanoma. These are particularly numerous when the primary melanoma shows features of regression.

Balancing extended sectioning against reverse transcription-PCR In the early stages of development of SLN pathology protocols, the possibility of using molecular techniques such as reverse transcription (RT)-PCR on fresh tissue instead of histology was considered. Positivity in SLN for melanoma using tyrosinase and or MART-1 or gp100 was found to be much higher than that obtained using histology, even up to $72 \%$ positive. ${ }^{16}$ This high positivity far exceeded what would be expected from our knowledge of the natural history of melanoma unless the selection of cases was highly selected in favour of extremely poor prognosis patients. However the comparison between histology and molecular techniques was not adequately controlled, most obviously in that the lymph nodes were divided in an unspecified way and the separate parts assessed by only one of the two techniques. This took no account of the distribution of the metastases in the nodes.

We decided that the role of molecular biology needed to be evaluated in a more controlled manner, ${ }^{14}$ and we found that a positivity of $45 \%$ with RT-PCR on frozen sections did exceed what was obtained from immediately adjacent sections stained conventionally (18\% positive), but that the discrepancy could be explained partly by the presence of naevus cells, which would also give a positive RT-PCR result, and partly by the presence of further melanoma deeper in the node only detected by more sections.

We achieved a detection rate of $33.8 \%$ by extending the sectioning protocol to six pairs of sections at $50 \mu \mathrm{m}$ intervals. Each pair was stained with $\mathrm{H} \& \mathrm{E}$ and S100. Other unstained spare sections were taken at each $50 \mu \mathrm{m}$ step. Further levels 
deeper than the sixth pair produced only a minimal improvement in detection rate and therefore the six pairs at $50 \mu \mathrm{m}$ intervals protocol was adopted by us as a reasonable balance between workload and sensitivity. ${ }^{14}$

The detection rate of $33.8 \%$ was still below $45 \%$ detected by RT-PCR but this is explained almost entirely by the presence of naevus cells. Others ${ }^{17}$ have suggested that this protocol is too labour intensive or expensive. More importantly the significance of the additional metastases needs to be established.

We do not regard the extra work as excessive since in more than $70 \%$ of SLN that are negative only seven slides need to be examined per lymph node. This is achieved by using S100 as a preliminary stain for scanning and one $\mathrm{H} \& \mathrm{E}$ to rule out other pathology. In the $30 \%$ of cases that are positive, more detailed assessment is required. Occasional melanomas are S100 negative ( $1 \%$ in our experience), but metastases are still identified easily as a negative or clear area against the blue counterstained background.

The only variation in the sectioning protocol occurs when lymph nodes are unusually rounded rather than approximately reniform. In those thicker lymph nodes we use a wider step between the pairs of stained sections, increasing it from $50 \mu \mathrm{m}$ to 75 or $100 \mu \mathrm{m}$.

It may be that the protocol we have adopted ${ }^{14}$ in the long term may be shown to be excessively rigorous since Van Akkooi et $a l^{18}$ have shown that metastases $<0.1 \mathrm{~mm}$ are not associated with further disease. However we consider that all metastases however small have yet to be shown not to be capable of progressing to more aggressive disease even after many years. We have some experience of metastases remaining small and localised in lymph nodes for several years after excision of the primary melanoma. This is presumably the explanation of how some melanoma metastases occasionally progress 10 or more years after excision of the primary. It remains to be shown whether some melanoma metastases are not capable of progression by reason of different biology related to their genetic profile, and therefore at least until that is proved and such cases are identifiable, detection of as many as possible of metastases should be the aim. This is supported by finding that so-called isolated tumour cells in groups less than $0.2 \mathrm{~mm}$ are associated with a lower overall survival. ${ }^{27}$

At least for the foreseeable future we recommend the protocol as outlined in table 1 using S100 as a screening stain so that the procedure is not too time consuming. RT-PCR is not ideal at least for detection of metastases but work of Hoon et a $l^{19}$ and Chew et al (unpublished) indicate that it may be useful in providing additional prognostic information.

\section{Naevus cells in SLN}

One of the main difficulties in interpretation of SLN is the presence of benign naevus cells. These are detected by S100 but are almost always negative with HMB45, so that marker can be used to distinguish naevus cells from metastatic melanoma. ${ }^{10}$ However, not all melanomas are positive with HMB45 (70\% in our experience) so that negativity is not a confirmation of benign nature.

More importantly, naevus cells can be distinguished from metastases by their site and cytology. We have assessed the SLN from 2005 patients with cutaneous melanoma, and 14\% of them contained naevus cells mostly in the substance of the fibrous capsule but occasionally in the fibrous trabeculae. The cells are usually characteristically naevoid with small dense nuclei or little cytoplasm. However, very occasionally, collections of naevoid cells are quite large so that they appear to encroach into the adjacent lymph node. In those cases, while nuclei are still highly uniform, nucleoli can become evident. In these rare difficult cases we use HMB45 (as recommended by Murray et $a l^{10}$ ) and MIB1. The HMB45 status is an important guide but sometimes a more definite conclusion is achieved with MIB1. Naevus cells should show no positivity with this stain, but melanoma would be expected to show at least some positive nuclei.

The interpretation of MIB1 can be difficult in lymph nodes because of the high proportion of positivity in surrounding lymphocytes. P16 can be used in a similar way being usually positive in naevus cells and lost in melanoma.

We have identified naevus cells in 279 SLN patients. Sixtytwo $(22.2 \%)$ of these were associated with melanoma metastases but the majority (217) were not.

\section{WHICH PATIENTS SHOULD BE OFFERED SLNB}

The criteria of the primary melanoma that are used to justify performing SLNB are not standardised but most commonly a minimum thickness of $1 \mathrm{~mm}$ has been used. Others have suggested that there is no benefit in performing SLNB on patients with melanomas thicker than $4 \mathrm{~mm}$ since these are already known to have a bad prognosis. However, we would argue that a large proportion (40\%) of the thick melanomas still have long-term survival and therefore the SLN can be used to attempt to identify that particular group. We originally considered that any patients with a melanoma in vertical growth phase should be offered SLNB and we did identify several patients with melanomas less than $1 \mathrm{~mm}$ down to $0.6 \mathrm{~mm}$ thickness that had metastasised. Nevertheless, the number of such cases was a small proportion of the total and therefore began to restrict offering SLNB to patients with melanoma thicker than $1 \mathrm{~mm}$. Gimotty et $a^{20}$ have suggested that thin melanomas $(<1 \mathrm{~mm})$ can be graded in risk according to their mitotic counts and the gender of the patient. Males with thin and invasive mitotically active melanomas are said to have a $31 \%$ risk of metastasis and females a $13 \%$ risk. Therefore we have begun to offer SLNB to patients with thin melanomas and dermal mitoses that by definition are in vertical growth phase.

Regression has also long been considered as an important feature of melanomas, with implications of an adverse effect on prognosis by some. This effect is not accepted universally and there are roughly equal numbers of studies who regard regression as having an unfavourable effect on survival as those who consider it to have no or even a favourable effect. ${ }^{21}$ Nevertheless prognostic tables ${ }^{22}$ showed regression as a poor prognostic factor and for that reason we did use it as a criterion for offering SLNB.

Our own studies ${ }^{23}$ comparing melanoma patients with or without regression in terms of SLN status, as a surrogate for survival, showed as one might expect intuitively that regression appeared to confer benefit to the patient, therefore we have discontinued accepting regression as a criterion.

Morton et $a l^{2}$ have identified some prolongation of diseasefree survival with SLNB on melanomas with thickness between 1.2 and $4 \mathrm{~mm}$ but this does not imply that SLNB should only be offered to patients with this range of thickness of melanoma since the staging benefits also apply to those outside this group.

At this stage we think it appropriate to offer SLNB to all patients with melanoma over $1.0 \mathrm{~mm}$ in thickness and to those patients with thinner invasive melanomas in which mitoses are identified in the dermal component unless there are other clinical features that override this decision. 
Table 1 Sequence of sectioning and staining for sentinel lymph node for melanoma

\begin{tabular}{ll}
\hline Section & Treatment \\
\hline Section 1 (first full section) & H\&E \\
Section 2 & S100 \\
Section 3 & Spare \\
$50 \mu \mathrm{m}$ gap $(+50 \mu \mathrm{m})$ & \\
Section 4 & H\&E \\
Section 5 & S100 \\
Section 6 & Spare \\
Section 7 & Spare \\
Section 8 & Spare \\
Section 9 & Spare \\
$50 \mu \mathrm{m}$ gap $(+100 \mu \mathrm{m})$ & \\
Section 10 & H\&E \\
Section 11 & S100 \\
Section 12 & Spare \\
$50 \mu \mathrm{m}$ gap $(+150 \mu \mathrm{m})$ & \\
Section 13 & H\&E \\
Section 14 & S100 \\
Section 15 & Spare \\
$50 \mu \mathrm{m}$ gap $(+200 \mu \mathrm{m})$ & \\
Section 16 & H\&E \\
Section 17 & S100 \\
Section 18 & Spare \\
$50 \mu \mathrm{m}$ gap $(+250 \mu \mathrm{m})$ & \\
Section 19 & H\&E \\
Section 20 & S100 \\
\hline In & \\
\hline
\end{tabular}

In large or round lymph nodes, the $50 \mu \mathrm{m}$ gap may be increased up to a maximum of $100 \mu \mathrm{m}$.

\section{MULTICENTRE SLN TRIALS}

The first multicentre SLN trial (MSLT-1) has made a preliminary report. ${ }^{2}$ In 1269 patients, this trial has shown that SLNB with immediate RND for positive cases had a $4.8 \%$ improvement in disease-free survival compared with no SLNB and RND only on clinical evidence of metastasis.

The SLN status was confirmed as a potent prognostic factor in that positive nodes conferred a 5-year survival rate of $72.3 \%$ on the patients compared with $90.2 \%$ in patients with negative SLN. No overall survival benefit was noted at 5-year follow-up. This does not exclude the possibility of survival advantage being demonstrated after longer follow-up since metastases from thinner melanomas tend to occur later if at all.

The second multicentre SLN trial (MSLT-2) proposes to evaluate whether it is necessary to proceed to RND in all patients with positive SLN irrespective of the tumour burden, and also to further examine the role of RT-PCR positivity in histologically negative SLN. Some cases that are histologically negative but positive on RT-PCR will still enter the trial.

The Sunbelt melanoma trial, which is another multicentre study, ${ }^{24}$ emphasises the prognostic and staging value of SLNB histology and also proposes to evaluate the role of RT-PCR in assessment of SLN, but has not yet concluded its study. It has reported a definition of SLN based on the ratio of radioactivity in a node to that in the background. The latter should be $10 \%$ or less of that in the SLN.

The Sunbelt trial has not identified factors in the SLN metastases that enable prediction of metastases elsewhere and therefore they advocate completion lymphadenectomy for every patient with a positive SLN. They have noted a lower rate of metastases to SLN in older patients despite the shorter survival in the same group, suggesting that the biology of melanomas of younger patients is often different from that of older patients, with the latter showing greater tendency to haematogenous metastases.

This is supported by the findings of Viros et al ${ }^{25}$ in which patients under 55 years were much more likely to have melanomas with BRAF mutation than older patients. This supports the growing support for the idea that melanoma is not one diagnosis but a collection of melanocytic tumours with different behaviours.

\section{A FUTURE ROLE FOR RT-PCR}

The MSLT-2 proposals have been stimulated in part by the observations of further RT-PCR studies ${ }^{19}$ that indicate that RTPCR positive, histologically negative SLN have a worse prognosis than those that are negative by both techniques. Our recent studies (unpublished) also suggest that nodal metastases detected by melanoma-specific markers such as MAGE-3 revealed by RT-PCR on paraffin-embedded tissue from SLN gives an added dimension to the prognosis beyond that which can be obtained with histology alone. However the process is laborious, therefore potentially expensive, and only dissects out an additional group of poorer prognosis patients from those $70 \%$ or so who are negative by histology. Rather than perform this additional assessment on the whole $70 \%$ of SLN that are negative, there is a need for a way of focussing on those within that group who are more likely to be positive. This would make this procedure more likely to be acceptable. For example, perhaps RT-PCR would be predictably negative in SLN-negative patients who were thinner than say $1.2 \mathrm{~mm}$ and had no mitoses in the dermis, or were of a histological type with suspected better prognosis such as spindle cell melanomas, or had a diffuse and intense tumour-infiltrating lymphocyte response.

Conversely SLN-negative cases that are histologically negative, but have a high mitotic count, would be expected to be more likely to be positive with RT-PCR.

\section{IMPROVEMENT OF PREDICTIVE ACCURACY OF HISTOLOGICAL ASSESSMENT OF SLN ACCORDING TO "TUMOUR BURDEN"}

A straightforward record of presence or absence of metastases in SLN has great prognostic value, ${ }^{1}$ but several authors have considered whether specific features of the metastases or their distribution may give more precise prognostic information. Starz et $a l^{11}$ described an index derived from the depth of the metastases from the capsule and the number of slices involved. This index enabled a prediction of the status of the rest of the lymph nodes in the same basin to be made with considerable accuracy. Cochran ${ }^{12}$ was able to provide increased accuracy of survival prediction by using a relative tumour area calculation, based on some computer-facilitated measurement and calculations.

Our group ${ }^{26}$ noted a correlation between the microanatomic distribution of the metastases in the SLNB and the presence of additional metastases in the RND. Those patients in whom the metastases were entirely subcapsular (fig 2) were found not to have any metastases in the nodes of the subsequent RND. The subcapsular pattern was seen in approximately $30 \%$ of all positive SLN. More recently Van Akkooi et al ${ }^{18}$ showed that metastases $<0.1 \mathrm{~mm}$ in maximum dimension, irrespective of site, were not associated with further metastases or any evidence of tumour progression. Govindarajan et al ${ }^{27}$ supported similar findings in noting that patients with metastases $<0.2 \mathrm{~mm}$ maximum dimension did not have positivity in their RND. There have been no recurrences in this group although 
the follow-up period was short. These findings require further study as the number of such cases that were not subcapsular was very small. In addition ${ }^{28}$ Scheri et al have noted that metastases $<0.2 \mathrm{~mm}$ in maximum dimension have a significantly higher risk of melanoma-specific death than SLNnegative patients thereby questioning the assumption that small metastases have limited significance.

These assessments of tumour burden all have merits, but whether one is more accurate or appropriate than another is not finally decided. All of them depend on careful examination of all available sections since metastases are not necessarily present in all. ${ }^{10}$ The Starz technique is perhaps most simple to assess being a measure from the capsule to the deepest metastasis. However one could ask from which part of the capsule does one measure. Logically it should be from the afferent aspect, but this is not always obvious.

Van Akkooi et al recommends the maximum dimensions of the largest deposit; this sounds straightforward but it needs to be understood that they mean the largest cohesive groups of cells, even though that may be only one of many, each of them showing only slight separation from each other.

The location of the metastases in our hands gives more reliable information, very easily assessed, in that subcapsular metastases are only very rarely associated with metastases in the rest of the nodes in the same basin. Even so, the definition of subcapsular site needs to be considered carefully. Subcapsular sinuses are not clearly visible and metastases in that zone are not usually identifiable as in a lumen. Their subcapsular zone location is important, with the configuration of the deposit being reasonably smooth, that is with no irregular projections on the parenchymal aspect.

On the basis of these studies it seems reasonable to presume that prognosis based on SLN can be refined, and that low volume, small or subcapsular metastases may not need to be followed by RND, although the criteria accepted may require further refinement. Whether these small or subcapsular metastases are precursors to larger tumours or represent a different character of metastases with more indolent growth properties remains to be established. It seems likely to us that

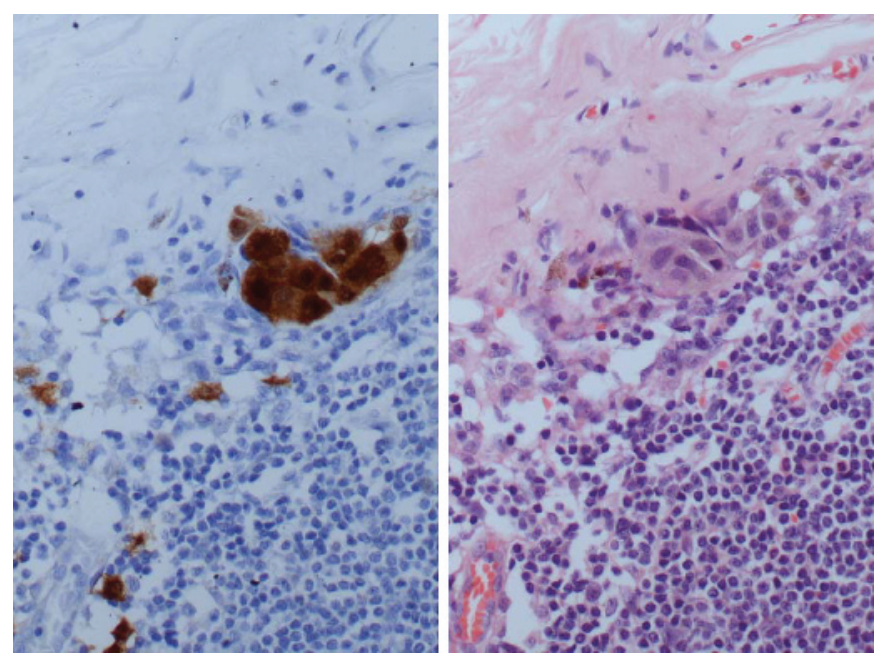

Figure 2 Screening of sentinel lymph node stained with S100 facilitates identification of metastatic melanoma. Distinction from dendritic cells, seen lower left, is made on the basis of cytology, with the dendritic cells having a poorly defined outline and smaller nuclei. The H\&E-stained adjacent section enables confirmation of the cytological features and the site of the metastasis-in this case subcapsular. they represent a mixture of early but potentially aggressive metastases with some more indolent ones.

In the meantime the European Organization for Research and Treatment of Cancer (EORTC) has suggested a clinical trial of melanoma patients with SLN metastases confined to the subcapsular zone or less than $0.1 \mathrm{~mm}$ in maximum dimension irrespective of site. These will be followed by observation for 5 years in the first instance. If these metastases are not associated with further disease then there would be some validation of a more selective approach to RND. It would also put more pressure on pathologists to assess the SLNB in greater detail. At the moment these assessments are performed only as part of studies or trials, but virtually all patients having SLNB are in that category since the procedure is to a large extent still being evaluated.

\section{SUMMARY: PART 1}

\section{Proposals for SLN assessment}

On the basis of our work and other published work we suggest that SLN for melanoma should be bivalved according to Cochran's method. The resulting paraffin-embedded blocks should be sectioned in our opinion in a stepwise manner.

We currently use $50 \mu \mathrm{m}$ steps producing six pairs of sections to an approximate total depth of $0.3-0.4 \mathrm{~mm}$. There is an argument to make the steps wider or less numerous, ${ }^{29}$ but bearing in mind the small size of many metastases and our lack of certainty on the critical dimension of the metastases we feel this change in protocol needs to be done in a systematic way. Van Akkooi et a ${ }^{18}$ suggest metastases $<0.1 \mathrm{~mm}$ diameter can be ignored. The EORTC observation study, in part, is intended to validate that observation and therefore we will continue with the existing EORTC protocol of six pairs of sections at $50 \mu \mathrm{m}$ intervals at least until that study reports.

We have strongly recommended the need for immunohistochemistry in the assessment partly because the detection rate is then higher and partly because the recognition of metastases is much facilitated, making the pathologist's task less onerous. Whether S100 or Melan A is used is a matter of personal choice. It is wise to retain some unstained sections at each level for problematic cases.

The features that should be recorded are not only the presence of metastases but their site and size.

A metastasis in a subcapsular site but with a markedly irregular aspect towards the parenchyma or single cells breaking away from the main metastasis should not be regarded as being entirely subcapsular in site.

Other measurements on which further refinements of management may be based are the depth of the metastasis from the capsule to its deepest point, and the maximum dimension of the largest deposit. Van Akkooi et al ${ }^{18}$ have suggested that this last dimension should be applied only to cohesive groups of cells rather than looser aggregates. Groups of 10 cells or less are already regarded by that group as negative. Others are taking a more cautious line and would prefer to await the results of more studies on this point. Since, at least in the UK, the SLNB procedure is not regarded as standard, and in all countries is being carefully evaluated, it is not suggested that the pathological assessments should simply be loaded onto pathology departments without careful consideration of the labour or cost implications. Furthermore, it is important that committed pathologists are involved in the reporting of SLN to ensure the reproductibility of the detailed assessments currently being undertaken in multicentre trials. 
We accept that SLNB for melanoma is in a phase of assessment and therefore we believe that the criteria we have set out and any new criteria need to be evaluated critically before discarding or adopting them formally as recommendations for general application.

\section{SUMMARY: PART 2}

\section{Probable further developments}

A lower threshold thickness of the primary melanoma for entry to SLNB could be considered as well as greater reliance on mitotic activity, particularly in males, and perhaps in due course on genetic profile. Since currently at least $70 \%$ of patients having SLNB have negative results, a greater focus on those who are more likely to be positive is predicted. This may be achieved by changes to the criteria of the primary melanoma used to justify performing SLNB.

It is also possible that RND will be offered to only those patients with metastases involving a parenchymal site or above a certain size.

There is some curiosity that some patients (30\%) with histologically negative SLNB nevertheless still develop recurrent disease and have a poor prognosis. Molecular studies may play a role at this point. Attempts will be made to predict who these patients are, using more specific markers.

If RT-PCR is adequately validated it is possible that the protocol for sectioning and staining could become less extensive. It seems likely that the need for S100 or Melan A will persist.

Other assessments may be needed since it is clear that the status of the immunological reaction to the metastases in the lymph node may be useful information when deciding upon immunologically mediated therapy. Dendritic cells are one component of this reaction that have been shown to correlate with survival. ${ }^{30}$ Tumour infiltrating lymphocytes (TIL) ${ }^{22}$ in the primary melanoma have prognostic significance in some studies. TIL in metastases may have a similar significance.

Irrespective of the controversy that SLNB seems to provoke it has stimulated much interesting analysis of the biological processes involved in metastasis, some of which may result in more beneficial therapy for the patients than the SLNB itself, and therefore it is likely to remain an important part of the management of melanoma patients for many years. It provides a major opportunity for pathologists to play a pivotal role in melanoma research and management.

Competing interests: None declared.

\section{REFERENCES}

1. Gershenwald JE, Thompson W, Mansfield PF, et al. Multi-institutional melanoma lymphatic mapping experience: the prognostic value of sentinel lymph node status in 612 stage I or II melanoma patients. J Clin Oncol 1999;17:976-83.

2. Morton DL., Thompson JF, Cochran AJ, et al. Sentinel-node biopsy or nodal observation in melanoma. N Engl J Med 2006;355:1307-17.

3. Morton DL, Cochran AJ, Thompson JF, et al. Sentinel node biopsy for early-stage melanoma: accuracy and morbidity in MSLT-I, an international multicenter trial. Ann Surg 2005;242:302-11.

4. Thomas JM, Clark MA. Selective lymphadenectomy in sentinel node-positive patients may increase the risk of local/in-transit recurrence in malignant melanoma. Eur J Surg Oncol 2004;30:686-91.

5. Estourgie SH, Nieweg OE, Kroon BB. High incidence of in-transit metastases after sentinel node biopsy in patients with melanoma. Br J Surg 2004;91:1370-1.

6. Kang JC, Wanek LA, Essner R, et al. Sentinel lymphadenectomy does not increase the incidence of in-transit metastases in primary melanoma. J Clin Oncol 2005;23:4764-70.

7. Pawlik TM, Ross Ml, Thompson JF, et al. The risk of in-transit melanoma metastasis depends on tumor biology and not the surgical approach to regional lymph nodes. J Clin Oncol 2005;23:4588-90.

\section{Take-home messages}

- Sentinel lymph nodes merit detailed histological assessment at several levels.

- Immunohistochemistry (S100 or MelanA) greatly facilitates the recognition of metastases of melanoma.

- Additional prognostic information can be obtained by assessing the sites and dimensions of metastases.

- Naevus cells occur in around $14 \%$ of sentinel lymph nodes and need to be distinguished from metastatic melanoma.

8. Staritt $\mathbf{E}$, Uren RF, Scolyer RA, et al. Ultrasound examination of sentinel nodes in the intitial assessment of patients with primary cutaneous melanoma. Ann Surg Oncol 2005; 12:18-23

9. National Institute for Health and Clinical Excellence. Improving outcomes for people with skin tumours including melanoma: The Manual. 22 February 2006. http:// www.nice.org.uk/guidance/index.jsp?action $=$ download\&o $=28906$ (accessed 2 June 2008).

10. Murray CA, Leong WL, Mccready DR, et al. Histopathological patterns of melanoma metastases in sentinel lymph nodes. J Clin Pathol 2004;57:64-7.

11. Starz H, Balda BR, Kramer KU, et al. A micromorphometry-based concept for routine classification of sentinel lymph node metastases and its clinical relevance for patients with melanoma. Cancer 2001:91:2110-21.

12. Cochran AJ. Surgical pathology remains pivotal in the evaluation of "sentinel" lymph nodes. Am J Surg Pathol 1999;23:1169-72.

13. Cochran AJ, Wen DR, Morton DL. Occult tumor cells in the lymph nodes of patients with pathological stage I malignant melanoma. An immunohistological study. Am J Surg Pathol 1988;12:612-8.

14. Cook MG, Green MA, Anderson B, et al. The development of optimal pathological assessment of sentinel lymph nodes for melanoma. J Pathol 2003;200:314-9.

15. Messina JL, Glass LF, Cruse CW, et al. Pathologic examination of the sentinel lymph node in malignant melanoma. Am J Surg Pathol 1999;23:686-90.

16. Bieligk SC, Ghossein R, Bhattacharya $S$, et al. Detection of tyrosinase mRNA by reverse transcription-polymerase chain reaction in melanoma sentinel nodes. Ann Surg Oncol 1999;6:232-40.

17. Scolyer RA, Thompson JF, Warnke K, et al. Pathology of melanocytic lesions: new controversial, and clinically important issues. J Surg Oncol 2004;86:200-11.

18. van Akkooi ACJ, de Wilt JHW, Verhoef C, et al. Clinical relevance of melanoma micrometastases $(<0.1 \mathrm{~mm})$ in sentinel nodes: are these nodes to be considered negative? Ann Oncol 2006;17:1578-85.

19. Hoon DS. Are circulating tumor cells an independent prognostic factor in patients with high-risk melanoma? Nat Clin Pract Oncol 2004;1:74-5.

20. Gimotty PA, Guerry D, Ming ME, et al. Thin primary cutaneous malignant melanoma: a prognostic tree for 10 -year metastasis is more accurate than American Joint Committee on Cancer staging. J Clin Oncol 2004;22:3668-76.

21. Cook MG. The significance of inflammation and regression in melanoma. Virchows Arch A Pathol Anat Histopathol 1992;420:113-5.

22. Elder DE, Murphy GF. Melanocytic tumours of the skin. Washington, DC: Armed Forces Institute of Pathology, 1991:163-4.

23. Kaur C, Thomas RJ, Desai N, et al. The correlation of regression in primary melanoma with sentinel lymph node status. J Clin Pathol 2008;61:297-300.

24. McMasters KM, Noyes DR, Reintgen DS, et al. Lessons learned from the Sunbelt melanoma trial. J Surg Oncol 2004;86:212-23.

25. Viros A, Fridlyand J, Bauer J, et al. Improving melanoma classification by integrating genetic and morphologic features. PLoS Med 2008;5:e120.

26. Dewar DJ,. Newell B, Green MA, et al. The microanatomic location of metastatic melanoma in sentinel lymph nodes predicts nonsentinel lymph node involvement. J Clin Oncol 2004;22:3345-9.

27. Govindarajan A, Ghazarian DM, McCready DR, et al. Histological features of melanoma sentinel lymph node metatases associated with status of the completion lymphadenectomy and rate of subsequent relapse. Ann Surg Oncol 2007;14:906-12.

28. Scheri RP, Essner R, Turner RR, et al. Isolated tumour cells in the sentinel node affect long term prognosis of patients with melanoma. Ann Surg Oncol 2007;14:2861-6.

29. Splanknebel K, Coit DG, Bieligk SC, et al. Characterisation of micrometastatic disease in melanoma sentinel lymph nodes by enhanced pathology: recommendations for standardising pathologic analysis. Am J Pathol 2005;29:305-17.

30. Elliott B, Scolyer RA, Suciu S, et al. Protective and long-term effect of mature DCLAMP+dendritic cell accumulation in sentinel lymph nodes containing micrometastastic melanoma. On behalf of the EORTC Melanoma Group. Clin Cancer Res 2007;13:3825-30. 